rattlesnake does not, however, prevent the entrance of the dog; the rattlesnake is never wanton, and only defends itself and takes necessary food. The dog will pass by it to enter its burrow without being molested.

Cleistogamous Flowers in Grasses.-Mr. C. G. Pringle has discovered in Western Vermont cleistogamous flowers in several grasses, especially Danthonia spicatc. The latter has many flowers totally concealed in the sheaths, the glumes and pales being nuch simplified, but the sexual parts being perfect and producing seeds. This plant is spreading rapidly in Vermont. The seeds borne on the top of the culn fall mostly at midsummer and lodge close to the parent plant, but the concealed seeds stored around the culm remain till these are disjointed and driven about by the autumn and winter winds; consequently, a wide means of dissemination is provided.

\section{ON THE VIEW OF THE PROPAGATION OF SOUND DEMANDED BY THE ACCEPT- ANCE OF THE KINETIC THEORY OF GASES}

1. $T^{T}$ is an accepted fact that the molecules of a gas are in motion among themselves in their normal state, and incapable of acting on each other at a distance; so that a theory of the propagation of sound, based upon the contrary suppositions that the molecules of a gas are at rest in their normal state and capable of acting on each other at a distance, cannot possibly be tenable. It thereby becomes necessary to inquire what view of the propagation of sound is demanded by the acceptance of the kinetic theory of gases; and this inquiry would appear to be all the more important in view of the fact that the mechanism of the propagation of sound in gases forms the physical basis of a great part of acoustics, or the groundwork upon which a number of its problems depend-the physical basis that underlies a system being admittedly the most important of the whole.

2. The molecules of a gas being in motion among themselves, it becomes evident after a very brief consideration of the question, that the only way in which a small impulse (or variation of velocity) termed a "wave" can be propagated through a gas, is by the exchange of motion normally going on among the molecules of the gas. For the molecules have no other mode of acting upon each other, excepting by exchange of motion. The rate at which this "wave" (or small variation of velocity) is propagated through the gas, will therefore depend on the rate at which the molecules cxcliange motion, i.e. on the normal velocity of the molecules of the gas. The sole condition determining the velocity of propagation of sound in a gas is therefore the velocity of the molecules of the gas. Here, therefore, we have a very simple condition for the velocity of sound (on the basis of the kinetic theory) or the velocity of sound becomes thus dependent only on one condition. This simplicity is characteristic of the rest of the kinetic theory, and is (it may be added) the recognised quality of scientific truth. In gases of the most diverse densities, specific gravities, pressures, and temperatures, the velocity of sound is only dependent on one condition, viz., the vilocity of the molecules, of the gas.

3. That the velocity of sound is independent of dersity, will be evident from the consideration that the molecules of gas are almost indefinitely small compared with their length of free path, and also the time of a collision is indefinitely small compared with the time taken to traverse the free path, so that it does not matter how many collisions (or exchanges of motion) occur along the line of passage of the impulse (or "wave"), but simply on the rate of motion of the molecules conveying the impulse. So (to take a simple analogy by way of illistration), it does not matter how many couriers are along the line of route conveying a message, but on the rate of motion of the couriers. Adding to the number of molecules in unit of volume of a gas (or adding to the density) does not, therefore, alter the velocity of sound in a gas, because it does not alter the velocity of the molecules which (by their exchange of motion) propagate the wave. The old theory supposes that the velocity of sound is here unaltered, because increased density diminishes the velocity of propagation of the wave, and increased pressure (attendant on the increased density) augments the velocity of the wave, and thus the two conditions counteract each other. On the kinetic theory, neither of these conditions can have any effect, and therefore the explanation of the unaltered velocity of the wave is perfectly simple, being the cosequence of the unaltered velocity of the molecules which propagate it. It is unnecessary to comment on the contrasted simplicity of the view on the kinetic theory; which is, moreover, the true view, if the kinetic theory be accepted.

4. That the velocity of sound on the kinetic theory is independent of pressure, is sufficiently clear at first sight ; for pressure evidently could not influence the rate at which the molecules exchange motion among each other, through which means alone the impulse is conveyed.

5. That change of specific gravity (or molecular weight) can by itself have no effect on the velocity of the sound-wave, is evident from the fact that it cannot matter whether the molecules exchanging motion among each other (and propagating the impulse) be heavy or light, provided their velocity be the same. It has been (as is known) demonstrated, generally from dynamical principles, that a system of bodies in free collision all tend to acquire the same absolute energy. Hence the velocity of each body depends on its mass (or varies inversely as the square root of its mass). So the mass of the molecules of hydrogen being (as is known) one sixteenth that of the molecules of oxygen, the velocity of the molecules of hydrogen is four times greater than that of the molecules of oxygen; and accordingly for this reason the velocity of sound in hydrogen is exactly four times greater than its velocity in oxygen-not, however, because the molecules propagating the wave are heavy or light. The molecules of hydrogen in their normal exchange of motion, move at four times the speed (compared with those of oxygen), and therefore propagate by this exchange of motion the sound-wave at four times the speed. The specific gravity (or molecular weight) of the gas has evidently nothing whatever to do with the rate of propagation of sound. The reason why the velocity of propagation of sound appears to depend on the molecular weight of the gas is because the velocity of the molecules of the gas depends on the molecular weight.

6. So also the velocity of sound is independent of the temperature, provided the molecular velocity remains the same. Of course this could only be true of different gases (i.e, of gases of different molecular weights), which-as is known-may be at different temperatures and yet possess the same molecular velocities. In one and the same gas of course the temperature could not be altered without altering the molecular velocity, for the "heat" itself consists in the motion of the molecules of the gas. This is therefore evidently the cause why the application of heat to a gas increases the velocity of sound. The addition of "heat" simply represents (as is known) the addition of velocity to the molecules of the gas, which consequently, by their exchange of motion, propagate the wave at a greater rate. The explanation of the increased velocity of sound in a heated gas is thus simple and direct. On the old theory the increased velocity of the sound-wave in a heated gas is referred to the diminished density of the heated gas (attendant on its expansion); and when the gas is confined, to its increased pressure, Surely this is at best a somewhat laboured and 
indirect way of accounting for a fact, and (as we have seen) according to the kinetic theory it cannot hold, since according to this theory, density and pressure can have no influence on the velocity of the wave, and on the other hand it is a known fact that the velocity of the molecules in their exchange of motion (by which means alone they can propagate the wave) is increased by the heat-indeed this augmentation of velocity itself represents the added "heat." This explanation of the increased velocity of a sound-wave in a heated gas commends itself therefore not only by its simplicity, but as a matter of scientific truth.

7. This serves also to explain in a direct and simple manner the relation the velocity of sound in a gas bears to the temperature. The absolute "temperature" of the gas represents (as is known) simply the energy of the molecules. The velocity of the molecules (as of any moving system of bodies) is proportional to the square root of their energy, and therefore proportional to the square root of the absolute temperature (since the "temperature " represents the energy). The velocity of sound, therefore (which is proportional to the velocity of the molecules), is thereby proportional to the square root of the absolute temperature of the gas.

8. To afford a more distinct idea of the mode of propagation of the wave and the physical effect (condensation and rarefaction) produced on the gas by its passage, the following considerations may serve. It is an important fact to keep in view that a system of bodies in free collision, such as the molecules of a gas, do not move in a mere chance or perfectly irregular manner, but a certain regularity exists. It has been mathematically proved that a forcible self-acting adjustment goes on among the colliding molecules of a gas so as to cause them to move in a special manner, viz., so that an equal number of molecules are moving in all directions, or as many molecules are moving in any one given direction as in the opposite. This mode of motion, if artificially disturbed, will correct itself. It is this special mode of motion (or movement of the molecules equally towards all directions) that produces the perfect equilibrium of pressure in all directions, observed in a gas.

9. From the fact that as many molecules are moving in any one direction as in the opposite; it follows that if an imaginary plane be placed in any position outside a vessel containing gas, the number of molecules (in the vessel) which at any instant are approaching the plane, is equal to the number which at the same instant are receding from it. Or otherwise, if we suppose any imaginary straight line in a gas, and visualise the molecules upon this line, then, as many molecules are moving in one direction as in the opposite. In the case of those molecules which are moving obliquely to the line, the resolved component of the motion in the direction of the line can be taken. This consideration enables the mode of motion of the molecules of a gas in its normal state, and the manner of propagation of waves through that mode of motion, to be illustrated in a very simple manner.

Io. In the annexed diagram, let $\mathrm{I}, 2,3, \& \mathrm{c}$., represent a line of spheres moving in such a way that as many spheres are moving in one direction as in the opposite. All the spheres marked with the odd numbers may be supposed

$$
\begin{array}{c|c:c:c}
\vec{\bullet} & \leftarrow & \bullet & \bullet \\
\hline & 2 & 3 & \bullet
\end{array}
$$

to move in one direction, while those marked with the even numbers move simultaneously in the reverse direction, the vis viva in the one direction balancing that in the opposite direction (as is the case with a gas). Each alternate sphere thus simply oscillates backwards and forwards in opposite directions within the limits represented by the dotted lines in the diagram, the spheres continually rebounding from each other, and the line of spheres tending to open out or expand and separate the final controlling surfaces $A$ and $B$ (like the expansive. action of a gas). It will be observed that this is in principle the only mode of motion possible by which the spheres can be in equilibrium; or half move in one direction and half in the opposite, so that the centre of gravity of the whole is at rest, in analogy with the centre of gravity of a portion of gas (the vis viva being at the same time balanced). There are only minute differences of detail as regards the comparison with a gas, none of principle. One detail is that every alternate molecule (in a line of molecules taken in a gas) does not necessarily move in an opposite direction, but it is rigidly true (on account of the vast multiplicity of molecules) that in any appreciable portion of a line taken in a gas, as many molecules are moving in one direction as in the opposite; for if not, the gas could not be in equilibrium in the direction of this line, whereas it is known to be in equilibrium in every direction. Another detail is that some of the molecules of a gas are moving obliguely to such an imaginary line, so that the mean path of the molecules is generally greater than that represented by the spheres. These details cannot however in the least affect the principle, and therefore the above method of illustration will serve (keeping in view the small differences mentioned) to convey a perfectly just idea of the character of the motion of the molecules of a gas in its normal state, and the way in which through that mode of motion "waves" are propagated through the gas. It is evident that an illustration is desirable in order to visualise clearly the facts. ${ }^{1}$

I I. Suppose, now, a slow oscillatory motion in the form of a movement of vibration to be communicated to the plane $A$. The plane $B$ may be supposed removed and the line of spheres extended indefinitely from the plane $A$. Then at the first forward swing of the plane $A$, the sphere $\mathrm{I}$ will receive an increment of velocity which it will transfer by collision to sphere 2 , the sphere I returning with its normal velocity to the plane, and receiving from it a second increment, \&c. By the forward swing of the plane, a succession of small increments of velocity will thus be propagated in the form of a pulse or semi-wave along the line of spheres, the velocity of propagation of the pulse being that of the spheres themselves. By the backward swing of the plane (to finish one complete vibration) a series of small decrements of velocity form. ing the second half of the wave will be propagated in precisely the same manner along the line of spheres. Owing to the succession of increments of velocity received by the spheres in the first half of the wave, these spheres will be shifted bodily forwards (to a slight extent), and owing to the succession of decrements of velocity sustained by the spheres in the second half of the wave, these spheres will be shifted (to a slight extent) bodily backwards, an alternate closing and opening out of the line of spheres corresponding to "condensations" and "rarefactions" being the result. There is only one slight (quantitative) difference in the case of an actual gas. Owing to the fact that some of the molecules in the case of a gas are moving (at the instant of passage of the wave) obliquely to the line of propagation of the wave, the rate of advance of the wave along the line of propagation will necessarily be somewhat slower than the velocity of the molecules which propagate it. It is (to take a homely illustration) as if some couriers were transmitting a message, and some of them were moving obliquely to the line of transmission of the message, wher evidently the rate of transmission would be less than the velocity of the couriers. In order to obtain the true rate of propagation of the wave in the gas, the oblique motions of the molecules must be taken into account.

12. In connection with a former paper ${ }^{2}$ bearing on this

I The mere fact of molecules, in the case of a gas, shifting their positions (through diffusion) can of course make no difference, since the saine character of motion is rigidly kept up. 
subject, the true mathematical expression for the velocity of the wave in terms of that of the molecules of the gas has been determined by Prof. Maxwell. The expression is Velocity of wave equals $\frac{\sqrt{5}}{3}$ into the velocity of the molecules. This expression requires a slight additional correction in the case of most gases, owing to the movements of rotation developed at the collisions of the molecules, depending on their more or less irregular shape, which rotation calculably must delay the wave to a certain extent. According to the experimental results of Kundt and Warburg, the above expression for the velocity of sound in terms of that of the molecules holds exactly true (without correction) for vapour of mercury (whose molecules, it might perhaps be remarked, are simple or monatomic). The slight deviations from the above constant for the velocity of the wave that one observes in fact, are quite consistent with what one would expect from theory.

13. It may be observed that all the usual apparatus for illustrating sound-waves of course applies to the kinetic theory, as such apparatus is only intended to show the effect produced on the mass of air, or the condensations and rarefactions, without exhibiting the molecular mechanism that underlies it. A true view of the mode of propagation of the wave and the manner in which the condensations and rarefactions are produced at its passage, can only be obtained by visualising the fact that the molecules of gas are in motion in the normal state of the gas, in accordance with the accepted kinetic theory of gases. ${ }^{2}$

14. The kinetic theory thus reduces the conditions on which the velocity of sound in a gas depends to one, viz., the velocity of the molecules of the gas. It is not, however, this simplification'alone that should recommend it, for it is not a mere question of choice or preference of one view over another, but a question of fact. For a theory of the conditions physically affecting the velocity of sound and its mode of propagation that may apply to one view as to the constitution of a gas (viz., the old view where the molecules are supposed at rest), cannot possibly apply to the diametrically opposite view of gaseous constitution represented by the accepted kinetic theory. It would appear desirable and fitting that the kinetic theory, having been applied so generally in other respects, should find a general application to so important and fundamental a fact affecting a gas as the propagation of sound in it.

15. Since the physical basis of a system is admittedly the most important of the whole, it would appear reasonable to expect that the investigation of problems in acoustics might gain by regarding the propagation of sound on the true physical basis represented by the accepted kinetic theory of gases; or by taking a true physical basis to ground the investigations upon, instead of one (based upon the old view of gaseous constitution) that admittedly does not harmonise with the facts.

NoTE.--It has recently come to my knowledge that two papers have been lately published on this subject, one by Prof. Roiti, of Florence (Nuoz'o Cimento, I877), the other by Prof. J. H. Hoorweg (Archiv Néer., xi., 1876), a brief abstract of which also appears in Beiblätter zu den Annalen der Physik und Chemie (vol. i. part 4, p. 209, 1877). Though the latter of these papers appears to precede mine (Phil. Mag., June, 1877), I may add that a sketch of the same theory appears in a little book ("Physics of the Ether," E. and F. N. Spon), published by $m e$ in 1875 . There is also an interesting paper by Mr. J. J. Waterston (Phil. Mag., 1859, supp. to vol. I6), in which he proposes to illustrate the propagation of

I It would appear not unreasonable to conclude that a realisation of the molecularbasis underlying the propagation of sound, according to the accepted kintetic theory, might be able to thruw some light on the investigations in connection with the telephine and other allied instruinents, where the mole. cular basis of the phenomena would seem to be the essential point to be considered. waves by a system of spheres, but he does not go into the explanation as to how the motion he assigns to the spheres can properly represent the case of a gas in its normal state. There are, nevertheless, points of interest in the paper.

\section{S. TOlver Preston}

\section{WHAT IS MORPHOLOGY $?^{1}$}

IF those of us who have laboured up the hill of life revert to the studies of our youth, I think we shall not remember to have heard our teachers speak of the "Morphology of Animals." I cannot remember when or where I first met with the word; although the idea itself with regard to plants, has been familiar to me for nearly forty years, that is, since the time when I became possessed of "Lindley's Introduction to Botany;" but he used the term "Organography." The term "Morphology" was used by Schleiden in his "Principles of Scientific Botany" at least thirty years ago; and I may say in passing that the study of that work was one of the best preparations I received for the work I have undertaken since.

A comparison of the mode in which both plants and animals are developed was suggested to me, if not for the first time, yet then with new force, by reading Johann Müller's "Physiology of Man ;" especially in the part on Generation, and more especially in his statement of, and criticisms upon, Caspar I. Wolff's "Theory of Generation," which was published at Halle in 1759 . The very mention of this date is interesting, for this is evidently the time, and this work of Wolff's was surely the work, which suggested to the great, rich mind of Goethe the idea of an underlying unity amid all the diversity of vegetable and animal forms. How fruitful this conception of the simplicity and unity of vegetable and animal patterns has been, I need not tell you; for more than a century it has been yielding precious and ever increasing results. It was natural, therefore, that a division of biology so new and so fascinating, should gain for itself a name : and as naturalists had been from time immemoria familiar with the metamorphosis of certain types, the term "morphology" which especially treats of such changes in the individual life-history of a plant or of an animal, was natural, easy, and appropriate.

The $a$ priori dreams which made the study of vertebrate morphology appear transcendental, and indeed gave it that title as a cognomen, caused great loss of time and of talent: and if Prof. Huxley had done nothing else whatever than dispel the glamour of these dreams, he would have deserved well of his age. His "Croonian Lecture," delivered at the Royal Society about twenty years ago, was as "a trumpet that gives a certain sound ;" the dreamers awoke from their dreams, and became the workers, who since that time have wrought with labour and travail night and day. But the science of morphology, which had become an elegant pastime here, had long before Prof. Huxley's time found a noble band of workers in Germany; from that land came the dream; in that land arose the workers; the labours of Rathke, von Baer, and Reichert were ready to the hand of our biological reformer. After these, who were the chiefs of the band, came others, all men of name and renown; "but they attained not to the first three."

My own indebtedness is primarily to Johann Müller, who in his "Physiology of Man," already referred to, gave such an excellent abstract of the labours of the embryologists, his countrymen. I ought not to forget his lamented translator, Dr, Baly; for in the original Müller's work was a sealed book to me, and indeed would be now.

The fact that all organic beings pass through various stages, and run a certain round of life, is now becoming

I The first of a course of lectures "s On the Motphology of the Batrachia," delivered at the Royal College of Surgeors, by Prof. W. K. Parker, F.R.S. 